\title{
EYE-SAFE FREE-SPACE LASER COMMUNICATIONS
}

\author{
by \\ Gary D. Wilkins \\ Wright Laboratory Avionics Directorate
}

\begin{abstract}
The Laser Communications Lab (LCL) of the Wright Laboratory Avionics Directorate is heavily involved in designing optical communications sysinvolved in designing optical communications syscommunications requirements. Due to the reluctance of many DOD agencies to adopt laser communications because of the potential eye-safety hazards associated with lasers, work is being accomplished in the LCL to develop an eye-safe laser communications system which will be powerful enough to provide communications over an extended range. This paper summarizes a system which has been designed, built and tested in the LCI using a prototype one watt continuous wave, two micron water diode Also summarized is the work accomplished using the Moderate Resolution Atmospheric Transmittance and Radiance Model, MODTRAN to choose the wavelength for the design.
\end{abstract}

Free-space laser communications is potentially one of the most versatile forms of communications available at the present time. Because of the narrow divergence of the laser beam, the communications channel has an inherent low probability of intercept (LPI) by those who are not the intended recipients. In addition, the short wavelength of the carrier produces a bandwidth which vastly exceeds our present capability to exhaust. Also, the channel is free from sources of electromagnetic interference which plague the radio frequency ( $\mathrm{rf}$ ) spectrum. Although guided laser communications using fiber optic cables has been around for years and the feasibility of using freespace laser communications for air-to-air applications was proven in the mid 1980's, there has been a reluctance by operational organizations in the military to accept laser communications has a source of LPI communications which can be used during RF commout scenarios. Much of this reluctance is based upon the fact that most laser communications equipment developed, up to this point, has not been eye-safe. This is a high priority requirement for those that need to maintain and use the equipment.

One other extremely important item to consider when using free-space laser communications in an atmospheric environment is the atmosphere. Whether communication is from air-to-air or air-to-ground, the atmosphere plays a major role in corrupting it.
Moisture and aerosols cause absorption and scattering losses, while temperature and pressure changes produce refractive index variations in the air by causing random variations in density. The result of these effects is a decrease of the transmittance of the atmosphere and a corresponding decrease in irradiance at the receiver.

As a consequence of an Air Force requirement for laser communications to be eye-safe, we launched an effort to study the eye-safe region of the infrared spectrum to determine its susceptibility to atmospheric turbulence and absorption, and to develop an eye-safe laser communications system. The wavelength region being explored is at two microns, which according to MODTRAN models is a window which can be exploited for long distance laser communications requirements. A two micron, one watt laser diode was purchased, and a communications system built and tested over our $8 \mathrm{~km}$ laser communications test range between the $\mathrm{LCL}$ Control/Receiver Facility (LCLCRF) in the WPAFB Area $B$ Building 620 Tower and the LCL Transmit Facility (LCLTF) at the WPAFB Trebein Road Facility (5) to gather propagation statistics.

The original objective of this in-house project was to test the performance characteristics of a commercially purchased 2.01 micron, diode pumped, solid-state laser to investigate the effects of atmospheric conditions on the quality of the received laser communications signal. However, the money was not available for the one watt diode pumped solid state 2.01 micron laser we wanted, and a one watt 2.01 micron laser diode was not available off-the-shelf, so we decided to purchase three prototype laser diodes from SDL. Early in the development of the prototypes, it became evident that they were not going to achieve the desired wavelength, so a great deal of atmospheric modeling was accomplished to provide SDL with an alternative wavelength which would allow us to have an eye-sale laser transmitter design close to the desired wavelength and communicate without excess atmospheric losses. Upon receipt of the laser diodes the laser transmitter and receiver were built and an eight kilometer laser communications link established between the LCLCRF and the LCLTF. 


\section{Eye-Safety}

There is a common misconception by engineers that wavelengths beyond 1.5 microns are completely eye-safe. The fact that these long wavelengths are not focused on the retina make them somewhat retina safe, in that energy is not concentrated on one spot such as the fovea, but does not make them totally eye-safe. The reason for this is that different wavelengths are treated differently by various parts of the eye. Some are absorbed by the Vitreous Humor in the eye and cause heating and painful swelling and drying, and some wavelengths are absorbed by other parts of the eye such as the lens or cornea and could cause permanent loss of vision. Given enough energy in the laser beam any laser wavelength could cause eye damage. The converse of this is that by restricting the amount of energy irradiated on the eye to a level below the Maximum Permissible Exposure (MPE) level as provided in American National Standards Institute (ANSI) Standard Z136.1-1993, any wavelength laser can be made eye-safe. The problem lies in the fact that with visible and near-infrared lasers, the MPE is very small for Nominal Ocular Hazard Distances (NOHD) which are close enough to the equipment to accomplish operational and maintenance procedures. Since there is a certain minimum amount of energy which is required to be radiated to provide an acceptable signal margin at the receiver, a communications transmitter using a visible or infrared wavelength will not be eye-safe unless the output radiated energy is either decreased, the divergence is increased or the transmitter exit aperture is increased. The first two of these approaches reduce the amount of energy at the receiver causing a decrease in the acceptable communication range, while the third requires an investment of more space at the transmitter to accommodate a larger aperture.

In an effort to determine the optimum laser specifications required to build the eye-safe laser communications transmitter, the Armstrong Laboratory LHAZ Version 2.0 Integrated Laser Hazard Assessment Program was used to generate MPE and NOHD data for a continuous wave (CW) 1 watt laser with a 1 centimeter aperture and divergence of 1 milliradian for $.25,1$, and 30000 second exposures for infrared wavelengths. Figures 1,3 , and 5 provide a graphical depiction of the MPE and figures 2, 4, and 6 illustrate the corresponding NOHD. As can be seen by Figure 2 , the eye-safe region starts at about 1.4 microns and a NOHD of 0 meters is required if the beam is only observed for .25 seconds. When the viewing time is increased to one second, even a 1 watt Carbon Dioxide laser with a wavelength of 10.6 microns and a divergence of 1 milliradian requires a NOHD of over 5 meters. For a person performing maintenance on such a transmitter that requires 8 hours of exposure an NOHD of 25.7 meters is required which means the operator had better be wearing eye and skin protection.

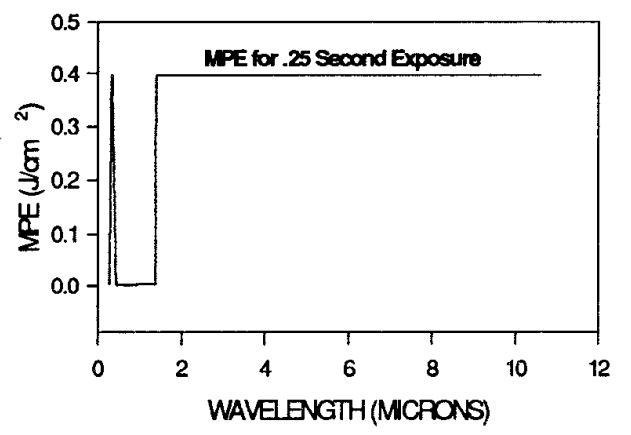

Figure 1 MPE for .25 Second laser Exposure

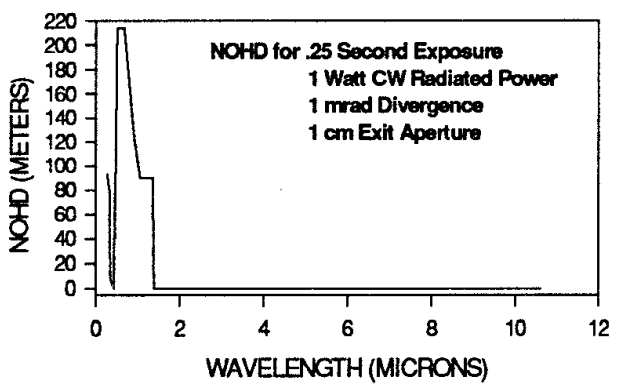

Figure 2 NOHD for .25 Second laser Exposure

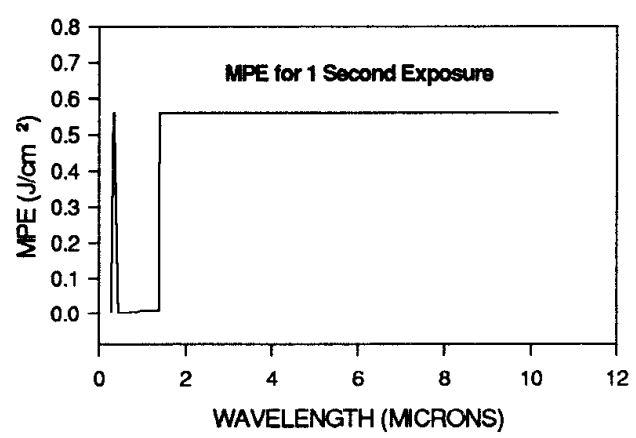

Figure 3. MPE for 1 Second Laser Exposure 


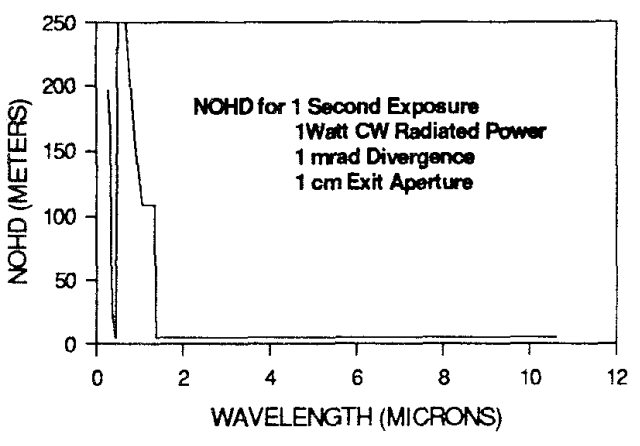

Figure 4. NOHD for 1 Second Laser Exposure

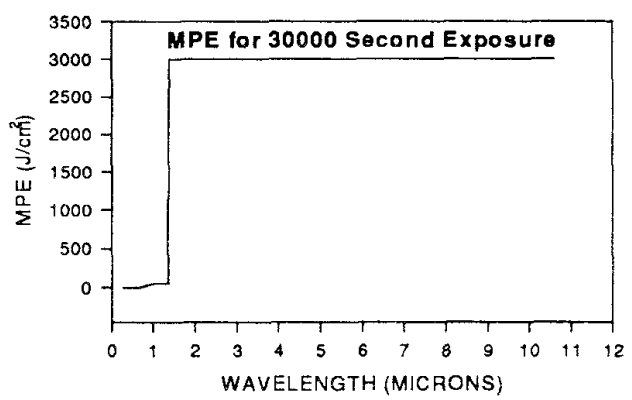

Figure 5. MPE for 30,000 Second laser Exposure

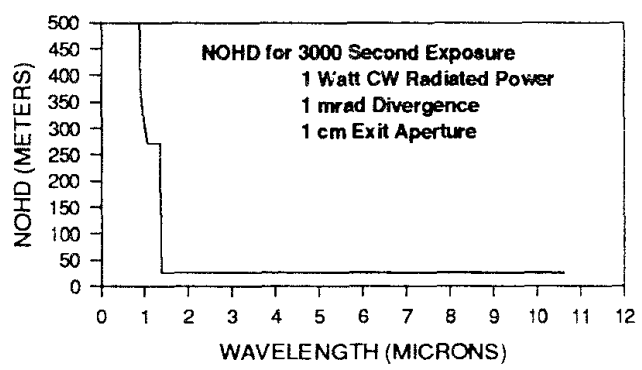

Figure 6. NOHD for 30000 Second Laser Exposure and $1 \mathrm{~cm}$ exit aperture

Since it was quite obvious from the NOHD that a 1 watt laser transmitter with divergence of $1 \mathrm{mrad}$ and an exit aperture of $1 \mathrm{~cm}$ was not eye-safe, the aperture setting of LHAZ 2.0 was increased in increments of $1 \mathrm{~cm}$ until the output of a 2 micron wavelength, 1 watt $\mathrm{cw}$ laser with a divergence of 1 mrad was eye-safe, i.e. the NOHD was 0 meters for a 30000 second exposure. This occurred at $4 \mathrm{~cm}$. The aperture size was then tweaked a little more until the smallest aperture which gave an NOHD of 0 meters was obtained. The result was an aperture of $3.58 \mathrm{~cm}$. Figures 7 through 9 provide the .25, 1, and 30000 second NOHD for the $3.58 \mathrm{~cm}$ exit aperture for the wavelengths used. From Figure 9 it is obvious that a 1 watt $\mathrm{CW}$ laser transmitter with a divergence of $1 \mathrm{mrad}$ and an exit aperture greater than $3.58 \mathrm{~cm}$ is eye-safe for any working distance over a period of 8 hours (the normal working man's workday).

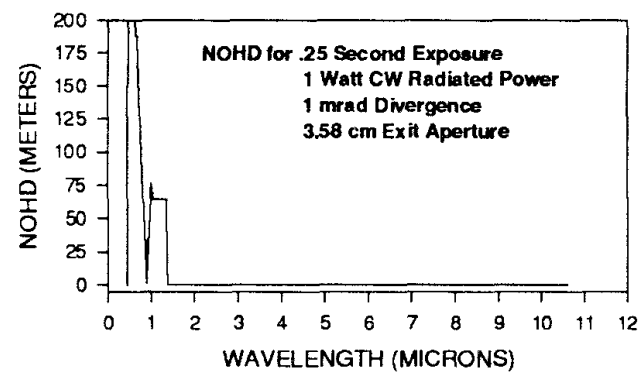

Figure 7. NOHD for .25 Second laser Exposure and 3.58 cm Exit Aperture

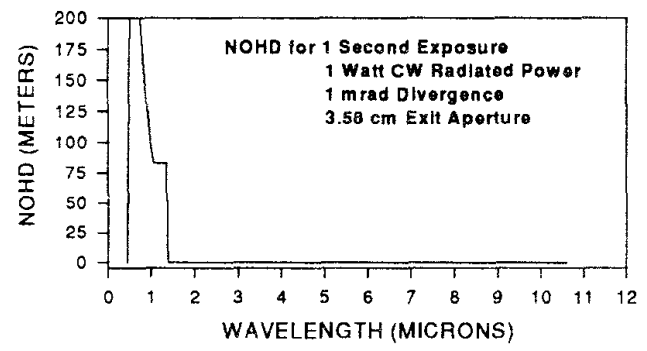

Figure 8. NOHD for 1 Second Laser Exposure and $3.58 \mathrm{~cm}$ exit aperture

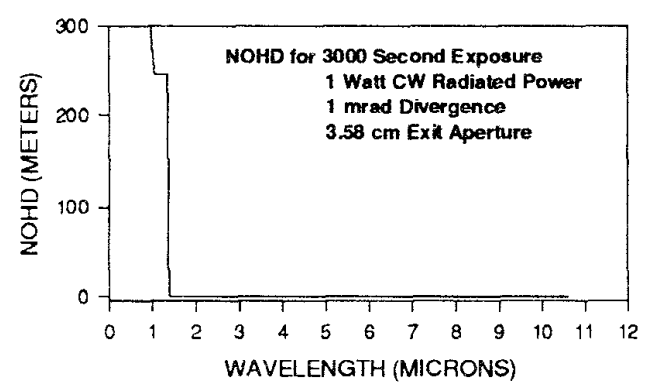

Figure 9. NOHD for 30,000 Second Laser Exposure and $3.58 \mathrm{~cm}$ Exit Aperture 


\section{Atmospheric Propagation Analysis}

No laser communications system design can be complete without knowledge of the conditions under which the system will be required to communicate. One large consideration is the atmospheric transmittance. In an effort to determine the best wavelength to use for our eye-safe laser communications system, we used the atmospheric modeling algorithm called MODTRAN to assess the transmittance of the atmosphere between the LCLCRF and LCLTF. We had originally requested a 2.01 micron laser because we knew of the exceptional transmittance from previous modeling exercises. When the Laser Manufacturer told us that they may not be able to reach the 2 micron goal for their 1 watt laser diode, we decided to use MODTRAN to determine the Transmittance of all Wavelengths between 1.4 and 2.1 microns to ascertain the best wavelength to shoot for if we had to settle for a shorter wavelength. Table 1 is a list of the parameters we used to accomplish our modeling.

\section{TABLE 1. TRANSMITTANCE MODEL INPUT DATA}

\author{
MODEL ATMOSPHERE - STANDARD MIDLATITUDE SUMMER \\ TYPE OF PATH - HORIZONTAL \\ PROGRAM EVALUATION MODE - TRANSMITTANCE \\ HAZE MODEL - AEROSOL ATTEN. FOR RURAL $23 \mathrm{KM}$. \\ SEASONAL PARAMETER - SPRING - SUMMER \\ VOLCANIC PARAMETER - STRATOSPHERIC BACKGROUND \\ CLOUD/RAIN PARAMETER - NO CLOUDS OR RAIN \\ ARMY VERTICAL STRUCTURE - NO \\ VISIBILITY - $23 \mathrm{KM}$ \\ RAIN RATE - 0 mm/HR \\ ALTITUDE OF SURFACE ABOVE SEA LEVEL - .330 KM \\ ALTITUDE OF TRANSMITTER ABOVE SEA LEVEL - .333 KM \\ ALTITUDE OF RECEIVER ABOVE SEA LEVEL - .366 KM \\ INITIAL ZENITH ANGLE - 90 DEGREES \\ RADIUS OF EARTH - 6371.23 KM \\ PATH LENGTH - $8 \mathrm{KM}$ \\ EARTH CENTER ANGLE - O DEGREES \\ INITIAL WAVELENGTH - $4000 \mathrm{CM}-1$ (2.5 MICRONS) \\ FINAL WAVELEN IN WAVENUMBERS $6666 \mathrm{CM}^{-1}(1.4 \mu \mathrm{m})$ \\ STEP SIZE - 1 CM- 1 \\ FULL WIDTH AT HALF MAX - 1 CM-1
}

As can be seen from the graphically depicted data in Figures 10 through 16, the best wavelengths to shoot for were in the range $1.97-2.0$ microns, with 1.9928 providing the best atmospheric transmittance. This information was provided to the laser manufacturer and three diodes were delivered in October 1994. The center wavelengths of the diodes included one at 1.992 and two at 1.988 microns, with each diode having about a 40 nanometer wavelength spread as shown in figure 17.

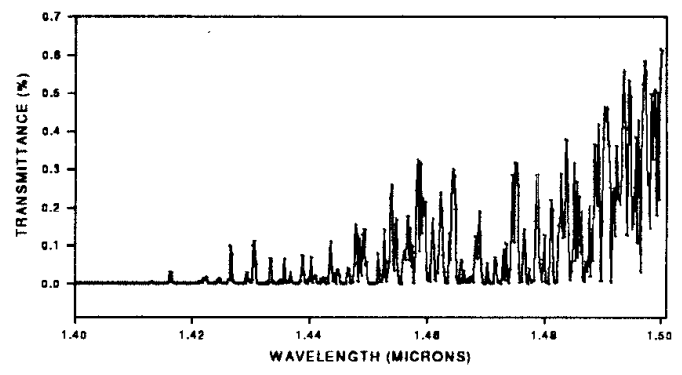

Figure 10. Atmospheric Transmittance Characteristics for 1.4 - 1.5 Micron Laser Wavelengths

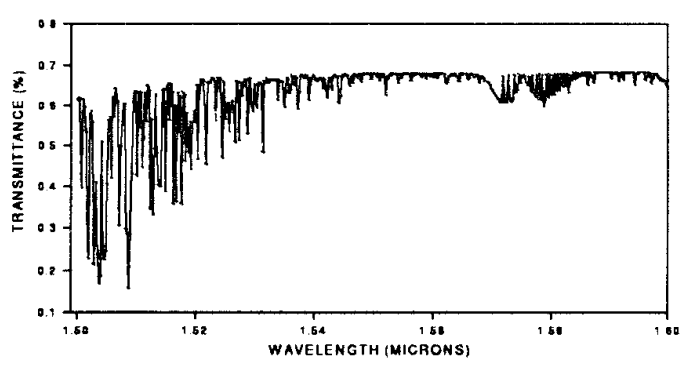

Figure 11. Atmospheric Transmittance Characteristics for 1.5 - 1.6 Micron Laser Wavelengths

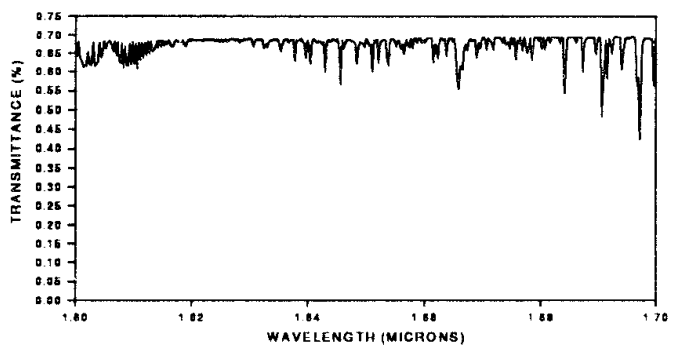

Figure 12. Atmospheric Transmittance Characteristics for 1.6 - 1.7 Micron Laser Wavelengths

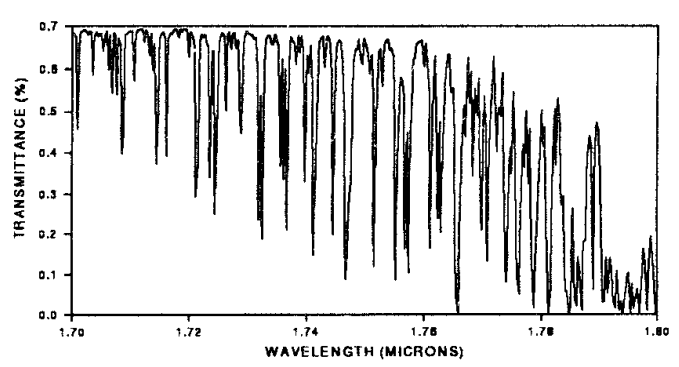

Figure 13. Atmospheric Transmittance Characteristics for 1.7 - 1.8 Micron Laser Wavelengths 


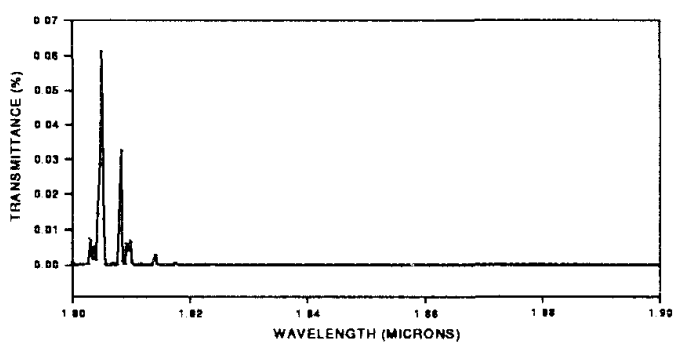

Figure 14. Atmospheric Transmittance Characteristics for 1.8 - 1.9 Micron Laser Wavelengths

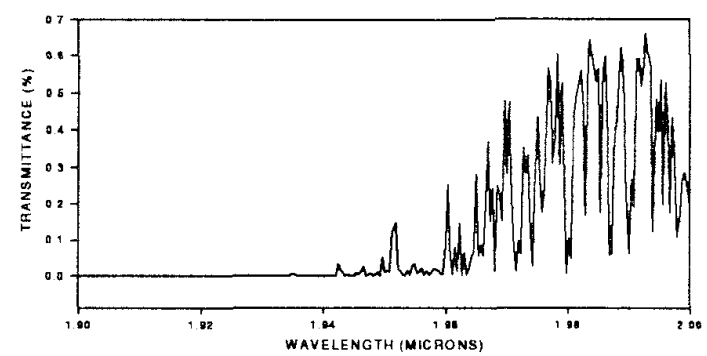

Figure 15. Atmospheric Transmittance Characteristics for 1.9 - 2.0 Micron Laser Wavelengths

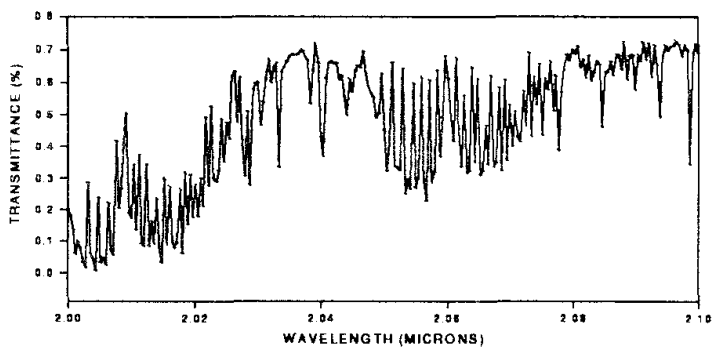

Figure 16. Atmospheric Transmittance Characteristics for 2.0 - 2.1 Micron Laser Wavelengths

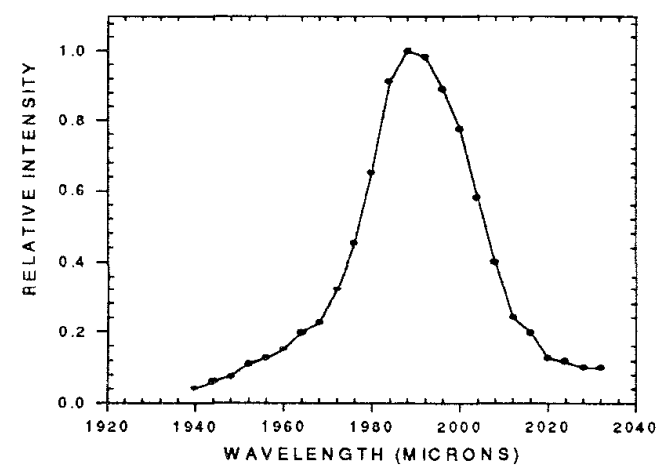

Figure 3-17. Spectral Characteristics of SDL One Watt $1.988 \mu \mathrm{m}$ Laser Diode
After the diodes were delivered with their specifications, MODTRAN was again utilized to determine the integrated transmittance over the wavelength range which each diode radiates at. An Initial wavelength of $4975 \mathrm{~cm}-1(2010 \mathrm{~nm})$ and a final wavelength of $5076 \mathrm{~cm}-1(1970 \mathrm{~nm})$ were used for the analysis. All other parameters in table 3-4 remained the same. The resulting transmittance was approximately $30 \%$. In other words, we are loosing more than two thirds of our signal to atmospheric absorption and scattering.

\section{Communications Equipment}

The equipment used for the data communications portion of the project consisted of the laser diode and power supply purchased from SDL, an in-house built receiver and off-the-shelf protocol analyzer equipment for measuring the quality of the communications link.

\section{Transmitter}

The laser transmitter used in the project was a Spectra Diode Laboratory AL944 laser diode mounted on a thermal-electrically cooled heatsink and powered by an SDL-820 laser diode driver and radiates 1 watt $\mathrm{CW}$ of optical output power. The transmitter is triggered by the return-to-zero TTL output of $a$ an RS232 -TTL interface connected to the RS232 output of an HP4957A protocol analyzer and emits a 40 nano-second pulse for each positive transition of the data input signal. Light radiated by the laser diode is collimated by a 3.58 inch diameter Infrared grade plano-convex lens. The maximum pulse repetition frequency of the transmitter has been determined by laboratory experimentation to be 16,800 pulses per second.

\section{Receiver}

The laser receiver was designed and built in-house. It consists of Celestron $90 \mathrm{~mm}$ Maksutov Cassegrain spotter scope adapted to focus the incoming laser radiation onto an Epitax corporation Indium Gallium Arsenide photodiode, housed with a bandpass filter in a Melles Griot modular photodetection system. A Stanford Reserch System Model SR530 Low Noise Current Amplifier supplies current to the detector and acts as the first stage amplifier. The signal is then amplified by a Stanford Research Systems Model SR560 low noise amplifier, which supplies the needed gain and electronic filtering. The resulting Gaussian shaped pulses are fed into the trigger input of Tektronix FG507 function generator where the leading edge of the pulse causes the function generator to output one TTL compatible pulse of preset duration for every Gaussian shaped pulse of a certain preset minimum amplitude at the input. The output of the 
function generator was subsequently fed into an HP 4957A Bit-Error-Rate Tester through a TTL-RS-232 converter for analysis.

\section{Bit-Error-Rate Testing}

Two HP 4957A Protocol Analyzers were used to measure the quality of the communications link. One HP 4957A was interfaced with the laser transmitter at the LCLTF and the second HP 4957A was interfaced with the in-house built receiver in the LCLCRF. Since the laser transmitter is edge triggered from the leading edge of a TTL pulse, an interface had to be built to convert the RS-232 Non-Return-to-Zero (NRZ) bit stream provided by the protocol analyzers to the TTL Return-to-Zero ( $R Z$ ) format required by the Transmitter. At the receiver, the received was then converted to NRZ and RS-232 format by an in-house designed and built TTL-to-RS232 converter. Synchronizing was not a big problem because the protocol analyzers were asynchronous. Although the sometimes low Bit-error-rate can probably be blamed on scintillation induced frequency jitter caused by a small diffraction limited aperture of the atmosphere (4).

\section{Observations and Conclusions}

The eye-safe laser communications system developed during this program is truly eye-safe as was shown by the LHAZ 2.0 results. The bit-error-rate approached a nominal $10^{-5}$ during periods of weather similar to the MODTRAN model discussed earlier. This is mainly due to the fact that the laser diode is a multi-wavelength device and some of the spectral bandwidth falls in the areas where absorption is high. The system signal margin could be increased by about $3 \mathrm{~dB}$, and the BER substantially reduced, by decreasing the laser diode spectral bandwidth to within just a few nanometers of the center wavelength. Further improvements in BER could also be achieved by implementing a synchronous modulation format.

\section{Future LCL Efforts}

The existence of the $L C L$ has been a tremendous asset in the research and development of past and present laser communications systems and will play an even more important role in the years to come as these developmental systems become operational. Plans are to accomplish projects to improve acquisition and tracking of laser communications signals, improve optical antenna designs to allow for near omni-directional coverage, and do further research into the role that atmospheric turbulence plays in corrupting the signal. Future laser communications development in the LCL will include the 1.54 to 1.68 micron region of the spectrum because work has already been accomplished to develop 1.54 micron laser diodes for the fiber optic industry and there are fewer absorption lines in that region to decrease the integrated transmittance over the 30 to 40 nanometer spectral range of the laser diode.

\section{Summary}

We set out in this project to develop a laser communications system which would be capable of operating in an atmospheric environment, over shortto-medium distances, and be eye-safe for operations and maintenance personnel. We did extensive analysis using the ANSI Standard and LHAZ 2.0 to ensure that the laser transmitter would be eye-safe over an entire 8 hour workday.

We have also attempted during this program to ascertain how free space laser communications using direct photodetection is affected by the presence of the atmosphere. A great deal of effort was expended in the early part of the program to select the optimum wavelength to use in our eye-safe laser communications system. By doing this we were able to design and build our system to meet the atmospheric challenges which our modeling had predicted. We hope to carry the process a step further and accomplish atmospheric turbulence modeling and testing to be able to gather enough insight into the problems of direct photodetection free space laser communications so we can develop an automated system which can adapt itself to obtain an optimum link in the presence of low atmospheric transmittance and high atmospheric turbulence.

\section{Bibliography}

1. Demers, H. , Feldmann; R.J., Fisher, K.; Holt, K.; Mercer, L.; Sebald, P. "HAVE LACE" AFWAL-TR-87-1128, Air Force Wright Aeronautical Laborities.

2. Wilkins, G.D. "Atmospheric Transverse Coherence Length Measurement System for Laser Communications", WL-TR93-1023, January 1996

3. Wilkins, G.D. " Eye-safe 2 micron Laser Communications System", WL-TR-96-1028, January 1996

4. Wilkins, G.D. "The Diffraction Limited Aperture of the Atmosphere and its Effects on Free Space Laser Communications, NAECON 92 Proceedings, Vol 3, May 1992

5. Wilkins, G.D. "The Wright Laboratory Avionics Directorate Laser Communications Laboratory, A Facility To perform Free Space Laser Communications System Testing", NAECON 94 Proceedings, Vol 1, May 1994

6. Wilkins, G.D. "Measurement of the Atmospheric Phase Coherence Length, $r_{0}$ " RADC-TR-86-192 December 1986 